\title{
Die Gesundheitskarte als Schlüssel zu eHealthcare
}

\author{
M. D. Denz ${ }^{a}$, G. C. von Below ${ }^{b}$
}

Um die Gesundheitsversorgung der Schweizer Bevölkerung mittels moderner Informations- und Kommunikationstechnologie (IKT) qualitativ verbessern zu können, sind nebst ethischen, juristischen und politischen Vorbedingungen eine Reihe primär technisch-organisatorischer Fragen zu lösen. Die elektronische Gesundheitskarte ist ein wichtiger Teil und Lösungsansatz.

\footnotetext{
a Medical Informatics/ eHealthcare FMH

b Medical Informatics/
} Quality Assurance FMH

1 Eine für die Verschlüsselung verwendete Zahlenfolge. Abhängig von dem eingesetzten Verfahren beträgt die Länge dieser Zahlenfolge zwischen 56 und mehreren tausend binären Stellen (Bits). Im Dezimalsystem entspricht dies einer Zahl mit 16 bis mehreren hundert Dezimalstellen.

Korrespondenz:

Dr. med. Martin D. Denz

Medical Informatics/

eHealthcare FMH

FMH

Elfenstrasse 18

CH-3000 Bern 16

\section{Identifikation der Teilnehmerinnen und Teilnehmer oder «Wer sieht was?»}

In der Gesundheitsversorgung ist die sichere Identifikation von natürlichen Personen von grösster Bedeutung; dies trifft sowohl für Patienten wie auch für Ärzte zu, und erst recht im Internet.

Der Mechanismus der Patientenidentifikation stellt einen integralen Teil des Behandlungsprozesses und der Patienteninformation dar. Die Methode zur eindeutigen Patientenidentifikation muss flächendeckend implementiert werden, um erfolgreich in den Behandlungsprozess integriert werden zu können. Dazu gehört zwingend auch die eindeutige Identifikation der Leistungserbringer.

Für sämtliche Interaktionen zwischen Patient und medizinischem Fachpersonal, welche über Computernetzwerke stattfinden, stellt die eindeutige Teilnehmeridentifikation eine unbedingt notwendige, aber nicht hinreichende Voraussetzung für eine sichere Kommunikation dar. Im Internetkontext ist keine implizite Identifikation (Stimme, Aussehen usw.) mehr möglich, so dass ein expliziter Authentisierungsprozess eingeführt werden muss. Diese Authentisierung beinhaltet auch einen administrativen Prozess, in dem die Beziehung zwischen dem zur Patientenidentifikation verwendeten Symbol auf einem sogenannten Token (z. B. SmartCard oder USB-Stick) bzw. der darauf enthaltenen Ziffernfolge, und dem Träger der Karte (z. B. Patient, Arzt, Apotheker) auf ihre Korrektheit geprüft wird.

\section{Verschlüsselung zur sicheren Datenübertragung}

Die Implementierung eines einheitlichen Identifikations- und Verschlüsselungssystems ist Voraussetzung für die Einführung der elektroni- schen Datenübertragung im Gesundheitswesen. Die im obigen Abschnitt beschriebene Basisfunktionalität zur Identifikation des Patienten oder Arztes (bzw. Spital, Labor usw.) bei der Übertragung von Patientendaten in elektronischer Form ist heute bereits in speziell gesicherten Netzen verfügbar (z.B. dem Health-Info-Net, http://www.hin.ch). Mittels digitaler Schlüssel ${ }^{1}$ und damit verbundener digitaler Zertifikate wird eine eindeutige, positive Identifikation (Authentisierung) erreicht, wobei die übermittelten Daten mittels einer sogenannten digitalen Signatur «unterschrieben» werden.

Digitale Schlüssel können ebenfalls dazu verwendet werden, alle übertragenen Daten so zu verschlüsseln, dass sie nicht verändert und nur für den Empfänger lesbar sind. So können sensible patientenbezogene Daten (Laborresultate, Überweisungsbriefe, Austrittsberichte usw.) sicher und gesetzeskonform elektronisch übertragen werden.

\section{«Patientenkarte» oder «Gesundheits- karte»?}

Die Begriffsverwendung spiegelt sowohl den technischen Entwicklungsstand als auch den beabsichtigten Verwendungszweck der Kartenlösungen. Kartenlösungen mit Magnetstreifen werden ihrer geringen Speichermenge wegen primär zu einfachen administrativen Zwecken (einfache Personalangaben), selten als «Notfallkarte» zur Speicherung minimaler Daten wie beispielsweise Blutgruppe und Allergien, eingesetzt (Problem: fehlende Standardisierung und Lesbarkeit). «Chipkarten» haben die Magnetstreifenkarten abgelöst und ermöglichen die Speicherung grösserer Datenmengen, verbunden mit rudimentären Kommunikationsfunktionen. Ihr Einsatz als «Patientenkarte» erfolgte mit der Absicht, möglichst grosse Datenmengen auf einer Karte zu speichern. Die fortschreitende Miniaturisierung führte zum Einbau «intelligenter» Mikrochips («SmartCards»), welche die Rechenstärke von Kleincomputern aufweisen. Dadurch eröffnet sich die Möglichkeit, mit einer Karte weit über die eigentliche Datenspeicherung hin- 
Abbildung 1

Schematische Darstellung eines elektronischen Patientendossiers.

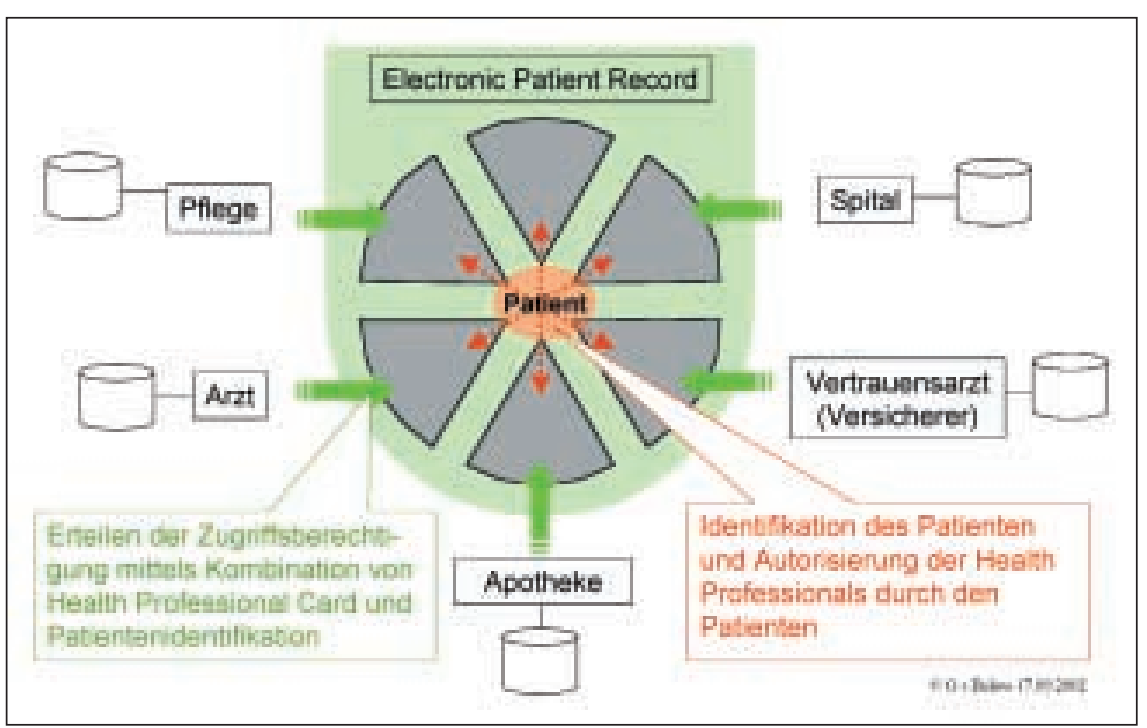

auszugehen und rechenintensive Verschlüsselungs- sowie Entschlüsselungsfunktionen auf einer Karte zu vereinen («Controller»).

Um Verwechslungen zwischen der Speicherfunktion und der Zugangsfunktion zu vermeiden, empfiehlt es sich, die Bezeichnung (elektronische) «Patientenkarte» für Karten zu verwenden, auf denen primär Patientendaten abgelegt werden sollen, quasi als elektronische «Mini-Krankengeschichte» oder «Datenbank im Kreditkartenformat». Für Karten mit einer (intelligenten) «Schlüsselfunktion» ist die Bezeichnung «Gesundheitskarte» geeigneter. Dabei ist zu beachten, dass die «SmartCard» für ihren Besitzer (= Patient) eine übergeordnete Funktion erfüllt, indem sie bei der Authentisierung von Health Professionals (z. B. Arzt) die komplementäre «health professional card» kontrolliert («Controller»-Funktion der Gesundheitskarte), über welche sich der Zugriffsberechtigte identifiziert hat.

Dieses komplexe Zusammenspiel ist Voraussetzung für den sicheren Austausch medizinischer Daten und deren Bearbeitung, beispielsweise zwischen Health Professionals, sowohl innerhalb von Institutionen als auch über grosse Distanzen wie z. B. das Internet.

\section{Einheitliches Datenmodell für elektronische Patientendaten}

Bei der Einführung von eHealthcare-Angeboten («Integrated Health Services») sind der Patient und sein Behandlungsprozess ins Zentrum zu stellen (sogenanntes Disease Management). Die elektronische Krankengeschichte bildet den funktionalen Kern sowohl für die Informationssysteme im Spital (Klinikinformationssysteme) als auch in der Praxis (Praxisinformationssysteme) (siehe Abb. 1).

Alle am Versorgungsprozess beteiligten Leistungserbringer und Institutionen legen die bei ihnen auf Papier oder elektronisch erfassten Patientendaten lokal ab. Lebenslang werden dadurch aus Sicht der Patienten dezentral verstreute Gesundheitsinformationen angelegt. Die sinnvolle Nutzung und der Austausch elektronischer Patientendaten zwischen Health Professionals ist nur mit einem einheitlichen Konzept zu gewährleisten [1]. Auch die Einführung und die Ausschöpfung der Möglichkeiten einer Gesundheitskarte ist nur möglich, wenn sie im Zusammenhang mit elektronischen Krankengeschichten eingesetzt wird [2]. Ein medizinischer, administrativer oder ökonomischer Nutzen der Gesundheitskarte muss durch eine gemeinsame Datenlogik, dem organisationsübergreifenden Datenmodell, und unter Verwendung einer gemeinsamen Sprache, von Kommunikationsstandards, ermöglicht werden [3].

\section{Gesundheitskarte und informationelles Selbstbestimmungsrecht}

Aus juristischer Sicht bestehen keine Zweifel: der Patient bestimmt künftig, wer zu welchem Zweck auf welche Informationen Zugriff haben darf. Eine Gesundheitskarte kann über die Grundfunktionalität des «digitalen Fingerabdrucks» (Identifikation und persönlicher Schlüssel) hinaus zur Verwaltung von Zugriffsrechten auf zentral oder dezentral gespeicherte Daten verwendet werden. Als Dateneigentümer wird der Bürger differenzierte Ebenen von Vertraulichkeit festlegen, wobei er Detaillierungsgrad und Einblicktiefe der am Versorgungsprozess beteiligten Institutionen und Personen auf seine Krankengeschichte seinen Bedürfnissen anpassen kann.

Auch die physische Lokalisation medizinischer Daten wird ihre Bedeutung verlieren. Die Patienten bzw. die von ihm mit Hilfe der Gesundheitskarte hierzu bevollmächtigten Health Professionals können diese «virtuelle Krankengeschichte» unabhängig von Ort und Zeit sogar über das Internet verwalten [4].

Bisher war die «Patientenkarte» Trägerin von «Krankheits»-Daten auf dem Weg vom einen zum nächsten Leistungserbringer. Der gesundheitsbewusste Bürger verfügt künftig mit seiner Gesundheitskarte über einen Controller, mit dem er aktiven Einfluss auf die Verwendung 
seiner persönlichen und medizinischen Daten nehmen kann. Dies ist auch im Lichte der sich weiterentwickelnden Patient-Arzt-Beziehung zu sehen [5]. Die Gesundheitskarte gewinnt dadurch an symbolischer Bedeutung: Gesunde und Kranke werden ihre Gesundheitsdaten selber «in die Hand nehmen» - auch die Verantwortung für ihre Gesundheit? [6, 7]

\section{Die Gesundheitskarte als Lösungsträger oder isoliertes Experiment?}

Der Entscheid des Bundesrats, der Einführung einer elektronischen Gesundheitskarte höchste Priorität zu geben, zeitigt erhebliche praktische Konsequenzen auf nationaler Ebene, welche viel weiter reichen als das Thema Datensicherung, oder der Zugriff auf Patientendaten, ebenso reichen sie über den Bereich Datenübertragung und den Datenaustausch hinaus [8].

Die Entwicklung einer einheitlichen elektronischen Krankengeschichte ist mehr als vordringlich, sie wird zum nationalen Notfall! Für den Spitalbereich, also «top down», wird in der Schweiz mit dem Projekt UNIT - Patientendossier $2003[9,10]$ Pionierarbeit geleistet.

Am Beginn des Versorgungsprozesses, also «bottom up», steht jedoch der Grundversorger meist noch ohne elektronische Krankengeschichte. In Sachen elektronisches Praxisinformationssystem ist die Schweiz ein Entwicklungsland, auch wenn wir aus den umliegenden Ländern auf viele (gute und schlechte) Erfahrungen zurückgreifen könnten [II]. Um der Gesundheitskarte eine Chance zu geben, brauchen wir die umgehende Erarbeitung von praxisorientierten Standards, d. h. Referenzmodelle in der Praxisinformatik (siehe [13]).

Parallel dazu laufen derzeit Bemühungen zur Implementierung einer für das ganze schweizerische Gesundheitswesen zuständigen Public Key Infrastructure (PKI) mit den entsprechenden Registrations- und Zertifizierungsstellen (Registration Authority bzw. Certificate Authority). Und nicht zuletzt muss auch der gesamten Bevölkerung eine geeignete Netzwerkinfrastruktur zur Verfügung stehen, auf der die (gesicherten) medizinische Daten national ausgetauscht werden können.

Die patientenorientierte und transdisziplinäre Bewältigung dieser Herausforderungen stellt einen wichtigen Schritt zur erfolgreichen Einführung von qualitativ hochwertigen, landesweit verfügbaren Informationssystemen und internetbasierten eHealthcare-Angeboten dar. Die Gesundheitskarte selbst wird ein Schlüssel zum
Erfolg dieser Angebote sein. Die an den Bedürfnissen der Endbenutzer/innen ausgerichtete Nutzung von Informationssystemen und «Integrated Health Services» wiederum bildet die Voraussetzung für die Akzeptanz der Gesundheitskarte.

Ganz entscheidend wird es somit sein, der Bevölkerung, den Health Professionals, Technikern und Politikern verständlich zu machen, dass der Erfolg von eHealthcare sich nicht automatisch aus Investitionen in primär technologische Lösungsansätze ergeben wird, sondern aus bedürfnisorientierten Projekten, welche den Kranken und Gesunden einen praktischen Nutzen eröffnen: Technologie als Lösungsträger, Technologie im Dienste des Menschen. [14]

\section{Literatur}

1 Kuhn KA, et al. From hospital information systems to health information systems. Problems, challenges, perspectives. Meth Inf Med 2001;40:275-87.

2 Grimson, et al. Delivering the electronic healthcare record for the 21st century. Int J Med Inform 2001;64:111-27.

3 McDonald CJ, et al. What is done, what is needed and what is realistic to expect from medical informatics standards. Int J Med Informatics 1998;48:5-12.

4 Masys D, et al. Giving patients access to their medical records via the internet. J Am Med Inform Assoc 2002;9:181-91.

5 Ball MJ, et al. E-health: transforming the physician-patient relationship. Int J Med Informatics 2001;61:1-10.

6 Cimino JJ, et al. What do patients do with access to their medical records? Medinfo 2001;1440-4.

7 Eysenbach $\mathrm{G}$, et al. The role of e-health and consumer health informatics for evidence-based patient choice in the 21st century. Clin Dermatol 2001;19:11-7.

8 Bates DW, et al. Reducing the frequency of errors using information technology. JAMIA 2001; 8:299-308.

9 http://www.sgmi-ssim.ch/bulletin/ B44_Ludwig.htm.

10 http://www.med-swiss.net/mitteilungen/ dateien/Patientendossier_Phase_3.pdf.

11 Mitchell E, Sullivan F. A descriptive feast but an evaluative famine: systematic review of published articles on primary care computing during 1980-97. Br Med J 2001;322: 279-82.

12 van de Lei, J et al. The introduction of computerbased patient records in the Netherlands. Ann Intern Med 1993;119:1036-41.

13 Zoller M, Wigger V, Bodmer U. Informatik in der ambulanten Medizin: Wo sitzt der Wurm? Schweiz Ärzezeitung 2002;83(39):2031-2.

14 Denz MD. «Neue Technologien»: mit E-health von High-tech zu High-touch. Schweiz Ärztezeitung 2001;82(42):2251-6. 